\title{
Comparison of the Structure, Regulation and Functions between Type Three and Type Six Secretion System in Gram-Negative Bacteria
}

Sara Badr ${ }^{1}$, Yanqi $\mathrm{Li}^{2}$ and Kangmin Duan ${ }^{1,2^{*}}$

${ }^{1}$ Department of Medical Microbiology, College of Medicine, Faculty of Health Sciences, University of Manitoba, Biology of Breathing Group, Children's Hospital Research Institute of Manitoba, 780 Bannatyne Ave, Winnipeg, MB R3E OW2, Canada

${ }^{2}$ Department of Oral Biology, College of Dentistry, Faculty of Health Sciences, University of Manitoba, Biology of Breathing Group, Children's Hospital Research Institute of Manitoba, 780 Bannatyne Ave, Winnipeg, MB R3E OW2, Canada

"Corresponding author: Kangmin Duan, Department of Oral Biology, College of Dentistry, Faculty of Health Sciences, University of Manitoba, Biology of Breathing Group, Children's Hospital Research Institute of Manitoba, 780 Bannatyne Ave, Winnipeg, MB R3E OW2, Canada, Tel: 2042733185; Fax: 2047893913; E-mail: Kangmin.Duan@umanitoba.ca

Rec Date: May 08, 2016; Acc Date: Nov 17, 2016; Pub Date: Nov 25, 2016

Copyright: (๑) 2016 Badr S, et al. This is an open-access article distributed under the terms of the creative commons attribution license, which permits unrestricted use, distribution, and reproduction in any medium, provided the original author and source are credited.

Abstract
Bacteria have evolved multiple protein secretion systems to survive and cope with surrounding environmental
stresses. So far, there are seven secretion systems (type I to type VII), which have been identified and demonstrated
the structural and molecular mechanisms. Among them, type three secretion system (T3SS), hallmark of acute
infection, is considered as the most complicated system and can translocate effector proteins directly into host cell
through a needle-like apparatus. Type six secretion system (T6SS) targets both prokaryotic and eukaryotic cells
using a bacteriophage-like structure and plays a role in pathogenesis and bacterial competition. This review is based
on our understanding of comparison of the structure, regulation, function and application between T3SS and T6SS.
Understanding the structural and functional mechanisms, as well as the difference and relationship between these
two secretion systems will help our understanding of bacterial pathogenesis and interspecies interaction.

Keywords: Secretion system; T3SS; T6SS; Antibacterial target

\section{Introduction}

Secretion systems have been developed by pathogens for the purpose of survival in host environments, competition, colonization, and infection. They facilitate the delivery of virulence factors (exoproteins-effector proteins-toxins) directly into the cytoplasm of target cells or into the environment [1]. So far considerable progress has been made towards elucidating the structural and functional mechanisms of the six secretion system (type I-VI) of gram-negative bacteria, the unique mycobacteria type VII secretion system. These systems differ from each other in the context of structure and functionality. Some toxins are secreted into the environment, whereas other toxins are secreted directly into the host cell to alter host physiology or for colonization [1]. In addition, these secreted proteins can be secreted through the inner and outer membrane in one step, such as type three secretion system (T3SS), T1SS, Type IV and Type VI. While, other proteins are secreted into the periplasmic space first through Sec or Tat pathways (two-step) then excreted to the outer membrane by T2SS, Type V or Type I and Type IV [2]. In the two-step secretion mechanism, Sec pathway is the most important export pathway. It is composed of Sec proteins, SecA (ATPase) and SecYEG. The other Tat (Twin Arginine Translocation) pathway is made of TatABC proteins and it is not considered essential as Sec [3,4]. T3SS is a needle like structure. It injects effector proteins directly into the cytoplasm of target host cells, to evade immune system and facilitate bacterial survival or to inhibit cellular function. T3SS is considered the most complex system among those six. It is present in animal and plant pathogenic or non-pathogenic symbiotic bacteria [5]. T6SS is one of the secretion systems recognized recently in 2006 in Pseudomonas aeruginosa and Vibrio cholerae. It is a one-step secretion system and it functions as a defense mechanism against other bacteria (antibacterial activity) in a polymicrobial environment. Type six secretion system (T6SS) along with type three secretion system (T3SS) are important for cell-cell interaction and virulence factors. Similarly, T6SS targets eukaryotic and prokaryotic cells. Its expression is controlled by several regulatory mechanisms such as $\mathrm{pH}$, quorum sensing, temperature change, two component regulatory systems, iron, magnesium and phosphate concentrations.

\section{Structure}

The structure of T3SS consists of a basal body, needle and translocon. There are several proteins which form or assist in forming the structure of the machinery, including PscC, PscN, PscF, PscP, PcrV, PopD, PopB [6]. The basal body is a ring-shaped body that consists of inner membrane (PscJ-lipoprotein) and outer membrane (PscC compounds). PscN is controlled by PscL and is thought to be an ATPase that fuels the system [7]. The needle is $60 \mathrm{~nm}$ to $100 \mathrm{~nm}$ in length, assembled by polymerization of PscF protein. Additionally, PscP protein determines and controls needle length [8]. It is believed that the needle plays a role in sensing host cell to activate T3SS [9]. A translocon/injectisome in T3SS is responsible for transporting effector proteins from the needle to the host cytoplasm [10]. PcrV hydrophilic protein is required for translocator assembly [11]. Permeabilization pore forming proteins PopD and PopB are hydrophobic translocator proteins, which function in host cells under acidic conditions (PH). Yet until this day, researchers are debating on the exact mechanism controlling needle length dimensions, and substrate switching. Bergeron et al and his group suggested a mechanism for sensing of needle length in T3SS through the ruler protein [12]. Ruler proteins are associated with the secretion of effector proteins in T3SS at early stages. Deletions of this protein results in aberrant needle length, and 
Page 2 of 4

the formation of multiple hooks in the flagellum. Studies on YscP ruler protein of Shigella T3SS revealed a conserved two domain structure. A length-sensing (LS) domain at the $\mathrm{N}$ terminal in charge of needle length, and a substrate-switching (SS) domain at the C terminal responsible for substrate switching through assembling T3SS structure and toxin secretion. Bergeron et al. reported that PscP ruler protein of Pseudomonas aeruginosa consists of a ball-and-chain structure revealing a disturbed LS and SS domain. Furthermore, they reported a crystal structure for the autoprotease PscU associated with LS domain. In addition, they demonstrated the structure of SS domain, and proposed that it is anchored to the tip of the needle due to its high stabilization. They hypothesized that the growth strength of the needle exerted on the SS domain leads to the detachment of the N-terminal anchor form the autoprotease. Thus, this mechanism allows for sensing of needle length by the ruler protein.

As for T6SS, there are three locus, H1-, H2-, and H3-T6SS. T6SS structure consists of a phage like tail with several subcomplex that secrete toxins into host cell/ bacteria in one step manner. T6SS harbors a gene cluster, which contain 13 genes essential for function, and encode proteins that form the system's structure. These proteins are named from TssA to TssM. Immunity proteins are encoded close to antibacterial effectors in T6S gene clusters to protect the secreting cell from its own toxins. TssJ, TssL, and TssM subunits make up the membrane anchoring complex, where the lipoprotein TssJ is anchored to the outer membrane. TssM is anchored in the inner membrane and have a c terminal region that interacts with TssJ. TssL is anchored in the inner membrane through a transmembrane helix, and interacts with TssM. The inner tube on T6SS is composed of Hcp, which is a protein related to gp19 in phage T4 [13]. The sheath is composed of contractile two proteins, TssB and TssC, which assemble in the cytoplasm to form tubular structures. VgrG a trimeric protein works on stacking Hcp hexamers and assembly of TssBC sheath. It also shares a similar structure with gp27/gp5 that form the tail spike in T4. PAARrepeat-containing proteins bind to VgrG protein. They particularly bind to the end of VrgG to form a sharp tip of Hcp-VgrG. Lastly, TssE protein forms the base structure of T6SS, and function as a platform for the bacteriophage like structure. It shares a homology with gp25 in T4, and is needed for the assembly of TssBC [14]. Moreover, TssAFGK proteins are suggested to form essential core components of the base [15]. For energy, a cytoplasmic AAA+ ATPase protein (ClpV) recycles the machinery after contraction by stimulating the disassembly of TssBC $[16,17]$. In addition, it can prevent and stop the polymerisation of the sheath structure. The whole mechanism of function in short consists of extension or assembly, contraction, and disassembly of the TssBC sheath. Some data suggest that T6S is only active efficiently on aggressive competitors with T6SS machinery. Tordello et al. were able to utilize type six secretion system sheaths as nanoparticles for antigen display [18]. T6SS sheaths are composed VipA and VipB proteins, which are associated with $\mathrm{ClpV}$. The group implemented these heterodimers $\mathrm{A}$ and $\mathrm{B}$ to produce immunogenic compound that can be used for vaccine delivery. Vibrio cholerae sheaths VipA and VipB fused with antigens were purified and prepared for immunization. They reported that enhanced immune response was observed against IgG in contrast to soluble antigens. Furthermore, serum bactericidal assay was implemented and confirmed the efficiency of antibodies against VipA IVipB. This application demonstrated the ability of T6SS sheaths to integrate various proteins in one nanoparticle, that can be transmitted on one antigen presenting cell (Figure 1).
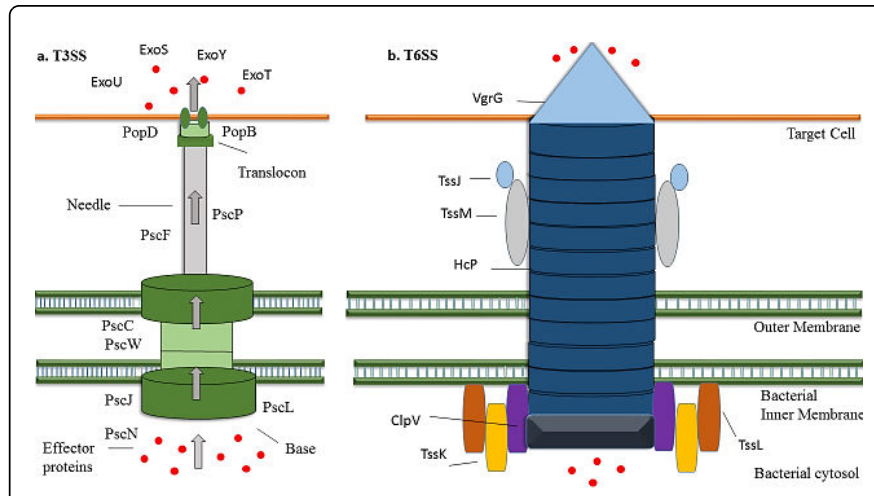

Figure 1: Structural organization of T3SS and T6SS in gramnegative bacteria.

\section{Function and Applications}

In case of T3SS, four effector proteins are secreted by T3SS in Pseudomonas aeruginosa, ExoS, ExoT, ExoY and ExoU. ExoS and $\mathrm{T}$ possess GTPase activating protein (GAP) activity and ADP-ribosyl transferase activity. ExoY is an adenylate cyclase and together with ExoS, ExoT they share a cytolytic activity and inhibit phagocytosis. ExoY also affects intracellular cAMP levels and cytoskeleton reorganization. Moreover, they are capable of disrupting endothelial cell junction by cell rounding to modify the cytoskeleton. Additionally, ExoS can inhibit DNA synthesis, cell to cell adhesion, and interact with $\mathrm{T}$ cells and monocytes resulting in T cell apoptosis. On the other hand, ExoT inhibits cell migration, and delay wound healing. Lastly, ExoU causes rapid necrotic cell death, and attacks neutrophils to increase the susceptibility of host immune system for secondary infections [19]. Studies indicated that T3SS alone without the secretion of effector proteins can actually trigger activation of caspase- 1 by the inflammasome via NLRC4 to cause cell death.

On the other hand, H1-T6SS secret six effector proteins that are responsible for bacterial competition. HSI-I also demonstrated a huge role in chronic infections in rat models of chronic respiratory infections, and in chronically cystic fibrosis patients. Studies have also demonstrated that T6S is capable of attacking eukaryotic cells. Whilst, $\mathrm{H} 2$ - and H3-T6SS play a double role by interacting with prokaryotic and eukaryotic cells. Deletion mutants were able to show attenuated H2-T6SS in mouse infections, and H3-T6SS displayed upregulation of gene clusters in the presence of epithelial cells. H2 interacts with bacteria in the environment by phospholipase D (PLD) PldA. Whereas, H3-T6SS-dependent PldB interacts with eukaryotic cells. These two effector proteins have also shown to degrade membrane phospholipids in Pseudomonas aeruginosa leading to its antibacterial activity, and promoting internalization into epithelial cells in humans. Furthermore, data from recent studies demonstrated that PldA and PldB activate PI3K-AKT signaling pathway to aid in invasion of epithelial cells. Effector proteins have been identified through bioinformatics, genetic and proteomics. First one to be identified was the cell wall targeting enzyme Tae (peptidoglycan amidase) as well as a Tge (peptidoglycan glycoside hydrolase). Another effector is Tle with proposed lipase/esterase activity. There are other effector proteins, which have not been identified yet, and others that have been identified, though their specific action is still unknown. Toxins are released into the target cell by the contraction of the sheath-like 
structure that spikes towards the cell. Furthermore, studies indicated that T6SS effectors can actually be translocated in two ways. They can be integrated with structural components and they are called specialised effectors. Or, they can interact with one of the essential components and these are called cargo effectors. In a recent study on type VI, Rogers et al. discovered that a protease LonA regulates biofilm formation, motility, virulence, and the type VI secretion system in Vibrio cholera. Proteases aid bacteria with adapting to environmental modifications, and protein degradation. Prokaryotic Lon plays a role in the degradation of foreign proteins, regulation of particular proteins, biofilm formation, motility, and virulence. A LonA mutant displayed increases swarming motility, alterations in cAMP levels, and biofilm formation in Vibrio cholera. Moreover, a whole genome expression demonstrated an increase in genes, which contribute to type VI secretion system when compared to wild type. Likewise, LonA mutant showed increased type VI efficiency, and deficient colonization in mice [20]. This result indicates the significance of LonA in the regulation of type VI system and virulence.

\section{Regulation}

Regulation of T3SS involves intrinsic and extrinsic regulation. It consists of different levels, transcriptional, post-transcriptional, and post-translational. The expression of effector protein is regulated by the major transcriptional activator ExsA in response to environmental signals [21]. These signals include direct contact with host cell, and low calcium. ExsA belongs to the AraC-family of DNA-binding proteins and it activates the transcription of T3SS through direct binding to promoters. Upon binding to the promoter, ExsA can facilitates transcription by enhancing RNA polymerase recruitment. A proteinprotein interaction network ExsCED, regulates ExsA at the posttranslational level. ExsC binds with ExsE in a 2:1 ratio and ExsD binds to ExsA [22,23]. Under inducing conditions, ExsE is secreted from T3SS and ExsC binds with ExsD in a 2:2 ratio. Thus, this binding with ExsD release ExsA and it can activate T3SS gene transcription. Extrinsic regulators are global regulators and include cAMP/Vfr, RsmAFYZ system, MucA/AlgU/AlgZR, and GacSA/RetS/LadS signaling cascade. T3SS gene expression have also been likened to quorum sensing. Recently, Marsden et al. discovered that virulence factor regulator (Vfr) can regulate expression of the Pseudomonas aeruginosa type III secretion system, by directly activating exsA transcription [24]. Vfr is a cAMP-dependent DNA-binding protein. It functions as a global regulator of virulence gene expression in Pseudomonas aeruginosa. In addition, it regulates type IV pili and type III secretion system. Marsden et al and colleagues reported that Vfr mutant displayed altered bistability, and analysed Vfr ability to regulated the transcription of the master regulator ExsA. They concluded that Vfr is capable of directly activating exsA transcription through a second promoter (PexsA) upstream of exsA PexsA promoter. This takes place upon the binding of Vfr to PexsA promoter probe, which was visualized by electrophoretic mobility shift assays. Furthermore, Vfr consensus-binding site was demonstrated by DNase I foot printing.

T6SS exhibits a complicated regulatory pathway in order to cope with surrounding stress in the unfavourable environment. Studies on the regulation of T6S showed that Gac/Rsm signalling is engaged in the activation of the system upon cell damage. RetS/LadS/GacS pathway regulates the expression of H1-T6SS in acute and chronic infections of Pseudomonas aeruginosa [25]. Regulation of T6SS is also associated with quorum sensing [26], iron uptake regulator [27], 854 - dependent activators [28], and a threonine phosphorylation pathway (TPP) in response to surface growth of the organism [29]. Interestingly, T3SS and T6SS can be switched by the sensor RetS via Cdi-GMP signalling [30].

\section{Conclusion and Future Prospects}

Bacteria delivers a series of effector proteins via different secretion systems to provide a fitness advantage in survival and competing for resources. Over the past few years, advances in structural and molecular biology have considerably improved our understanding of bacterial T3SS and T6SS and have led to the emergence of new drugdesign efforts. We can use the central regulator of each system, like exsA-master regulator of T3SS; core component of secretion system, like ClpV in T6SS; both regulators of these two secretion systems, like quorum sensing, iron uptake regulator.

Researchers are continuously trying to find new antimicrobial therapeutic targets against these secretion systems. For instance, they have targeted effector proteins, structure components, transcription pathways, regulatory genes, as well as inhibition of their assembly and action. Yet, there is still more about these systems to be understood when it comes to characterization regulatory genes, studying the interactions in gene network, and integral function of effector proteins interaction. Even though we have made great progress on these two secretion systems, there are many questions still unanswered. For example, what detailed environmental signals specifically trigger different secretion system? And how? Which regulatory networks influence T3SS/T6SS expression? What kind of regulators and signals are responsible for the activation of T3SS/T6SS regulatory system? Answering these questions still need some years and such detailed insight are needed to reveal new potential drug targets and to identify new directions for the development of novel anti-virulence compounds.

\section{References}

1. Gerlach RG, Hensel M (2007) Protein secretion systems and adhesins: The molecular armory of Gram-negative pathogens. Int J Med Microbiol 297: 401-415.

2. Palmer T, Berks BC (2012) The twin-arginine translocation (Tat) protein export pathway. Nat Rev Microbiol 10: 483-496.

3. Tseng TT, Tyler BM, Setubal JC (2009) Protein secretion systems in bacterial-host associations, and their description in the Gene Ontology. BMC Microbiol 9 Suppl 1: S2.

4. Rego AT, Chandran V, Waksman G (2010) Two-step and one-step secretion mechanisms in Gram-negative bacteria: Contrasting the type IV secretion system and the chaperone-usher pathway of pilus biogenesis. Biochem J 425: 475-488.

5. Bleves S, Viarre V, Salacha R, Michel GP, Filloux A, et al. (2010) Protein secretion systems in Pseudomonas aeruginosa: A wealth of pathogenic weapons. Int J Med Microbiol 300: 534-543.

6. Lara-Tejero M, Kato J, Wagner S, Liu X, Galán JE (2011) A sorting platform determines the order of protein secretion in bacterial type III systems. Science 331: 1188-1191.

7. Yip CK, Kimbrough TG, Felise HB, Vuckovic M, Thomas NA, et al. (2005) Structural characterization of the molecular platform for type III secretion system assembly. Nature 435: 702-707.

8. Marlovits TC, Kubori T, Tejero ML, Thomas D, Unger VM, et al. (2006) Assembly of the inner rod determines needle length in the type III secretion injectisome. Nature 441: 637-640.

9. Marlovits TC, Kubori T, Sukhan A, Thomas DR, Galán JE, et al. (2004) Structural insights into the assembly of the type III secretion needle complex. Science 306: 1040-1042. 
Citation: Badr S, Li Y, Duan K (2016) Comparison of the Structure, Regulation and Functions between Type Three and Type Six Secretion System in Gram-Negative Bacteria. J Med Microb Diagn 5: 243. doi:10.4172/2161-0703.1000243

Page 4 of 4

10. Galan JE, Wolf-Watz H (2006) Protein delivery into eukaryotic cells by type III secretion machines. Nature 444: 567-573.

11. Costa TR, Felisberto-Rodrigues C, Meir A, Prevost MS, Redzej A, et al. (2015) Secretion systems in Gram-negative bacteria: Structural and mechanistic insights. Nat Rev Microbiol 13: 343-359.

12. Bergeron JR, Fernández L, Wasney GA, Vuckovic M, Reffuveille F, et al. (2016) The Structure of a Type 3 Secretion System (T3SS) Ruler Protein Suggests a Molecular Mechanism for Needle Length Sensing. J Biol Chem 291: 1676-1691.

13. Miyata ST, Bachmann V, Pukatzki S (2013) Type VI secretion system regulation as a consequence of evolutionary pressure. J Med Microbiol 62: 663-676.

14. Leiman PG, Basler M, Ramagopal UA, Bonanno JB, Sauder JM, et al. (2009) Type VI secretion apparatus and phage tail-associated protein complexes share a common evolutionary origin. Proc Natl Acad Sci U S A 106: 4154-4159.

15. Cianfanelli FR, Monlezun L, Coulthurst SJ (2016) Aim, load, fire: The type VI secretion system, a bacterial nanoweapon. Trends Microbiol 24: 51-62.

16. Basler M, Pilhofer M, Henderson GP, Jensen GJ, Mekalanos JJ (2012) Type VI secretion requires a dynamic contractile phage tail-like structure. Nature 483:182-186

17. Kube S, Kapitein N, Zimniak T, Herzog F, Mogk A, et al. (2014) Structure of the VipA/B type VI secretion complex suggests a contraction-statespecific recycling mechanism. Cell Rep 8: 20-30.

18. Del Tordello E, Danilchanka O, McCluskey AJ, Mekalanos JJ (2016) Type VI secretion system sheaths as nanoparticles for antigen display. Proc Natl Acad Sci U S A 113:3042-3047.

19. Woods DE (1987) Pathogenesis of acute and chronic Pseudomonas aeruginosa infections. Antibiot Chemother (1971) 39: 160-171.

20. Rogers A, Townsley L, Gallego-Hernandez AL, Beyhan S, Kwuan L, et al. (2016) The lonA protease regulates biofilm formation, motility, virulence, and the type vi secretion system in Vibrio cholerae. J Bacteriol 198: 973-985.
21. Zheng Z, Chen G, Joshi S, Brutinel ED, Yahr TL, et al. (2007) Biochemical characterization of a regulatory cascade controlling transcription of the Pseudomonas aeruginosa type III secretion system. J Biol Chem 282: 6136-6142.

22. Diaz MR, King JM, Yahr TL (2011) Intrinsic and Extrinsic Regulation of Type III Secretion Gene Expression in Pseudomonas Aeruginosa. Front Microbiol 2: 89.

23. Izore T, Job V, Dessen A (2011) Biogenesis, regulation, and targeting of the type III secretion system. Structure 19: 603-612.

24. Marsden AE, Intile PJ, Schulmeyer $\mathrm{KH}$, Simmons-Patterson ER, Urbanowski ML, et al. (2016) Vfr Directly Activates exsA Transcription To Regulate Expression of the Pseudomonas aeruginosa Type III Secretion System. J Bacteriol 198: 1442-1450.

25. Silverman JM, Brunet YR, Cascales E, Mougous JD (2012) Structure and regulation of the type VI secretion system. Annu Rev Microbiol 66: 453-472.

26. Hense BA, Kuttler C, Müller J, Rothballer M, Hartmann A, et al. (2007) Does efficiency sensing unify diffusion and quorum sensing? Nat Rev Microbiol 5: 230-239.

27. Sana TG, Hachani A, Bucior I, Soscia C, Garvis S, et al. (2012) The second type VI secretion system of Pseudomonas aeruginosa strain PAO1 is regulated by quorum sensing and Fur and modulates internalization in epithelial cells. J Biol Chem 287: 27095-27105.

28. Bonemann G, Pietrosiuk A, Diemand A, Zentgraf H, Mogk A (2009) Remodelling of VipA/VipB tubules by $\mathrm{ClpV}$-mediated threading is crucial for type VI protein secretion. EMBO J 28: 315-325.

29. Schlenker C, Surawicz CM (2009) Emerging infections of the gastrointestinal tract. Best Pract Res Clin Gastroenterol 23: 89-99.

30. Moscoso JA, Mikkelsen H, Heeb S, Williams P, Filloux A (2011) The Pseudomonas aeruginosa sensor RetS switches type III and type VI secretion via c-di-GMP signalling. Environ Microbiol 13: 3128-3138. 